\title{
Hospitalized cancer patients with acquired long QT syndrome-a matched case-control study
}

\author{
Yajuan Lin ${ }^{1+}$, Haixu Yu ${ }^{2+}$, Fei Liu', Cheng Chen', Yanli Zhang ${ }^{1}$, Binhao Wang ${ }^{3}$, Yiheng Yang ${ }^{1}$, Ying Liu', \\ Li Zhang ${ }^{4,5^{*}}$ and Yunlong $\mathrm{Xia}^{{ }^{*}}$
}

\begin{abstract}
Background: Our recent study has revealed that many hospitalized patients with acquired long QT syndrome (ALQTS) are cancer patients. This study aims to determine the risk factors and outcomes of hospitalized cancer patients with ALQTS.

Methods: We performed a matched case-control study within a cohort of 10,180 cancer patients hospitalized between September 2013 and April 2016. Among them, 150 patients defined as having severe ALQTS with a markedly prolonged QT interval (QTc $\geq 500 \mathrm{~ms}$ ) were compared with 293 age-, sex- and cancer-type-matched controls (non-ALQTS). Death as the endpoint was followed for up to 2 years. Cox regression and Kaplan-Meier survival analyses were performed to assess the effects of particular clinical variables on all-cause mortality. Multivariate logistic regression was performed to calculate odds ratios (OR) for various predictors of QT prolongation.

Results: The mortality was significantly higher in ALQTS group (63.3\% vs. 33.4\%). Hypertension, hypokalemia, hypocalcemia, QT-prolonging drugs, infection, anemia, anti-microtubule agents were contributing factors to ALQTS. Renal insufficiency, male gender and hypokalemia were found to be independent risk factors for allcause mortality in ALQTS group.

Conclusion: Markedly prolonged QT interval was seen in 1.5\% of hospitalized cancer patients. The all-cause mortality was high in cancer patients with severe ALQTS.
\end{abstract}

Keywords: Acquired long QT syndrome, Cancer, Cardiotoxicity, All-cause mortality

\section{What's new}

Cardiotoxicity related to cancer therapy is of rising concern since it negatively impacts the prognosis. The 2016 European Society of Cardiology guideline described updated standard therapies for acquired long QT syndrome (ALQTS) associated with cancer therapy. However, evidence-based data regarding ALQTS in hospitalized cancer patients is limited.

\footnotetext{
* Correspondence: Idlzhang@gmail.com; yunlong_xia@126.com

${ }^{+}$Yajuan Lin and Haixu Yu contributed equally to this work.

${ }^{4}$ Sidney Kimmel Medical College of Thomas Jefferson University, Philadelphia, PA, USA

'Department of Cardiology, First Affiliated Hospital of Dalian Medical University, Zhongshan Road 222, PO Box 116011, Dalian, Liaoning, China Full list of author information is available at the end of the article
}

\section{Clinical implications}

In our study cohort, markedly prolonged QT interval was seen in $1.5 \%$ of hospitalized cancer patients. The all-cause mortality was high in cancer patients with severe ALQTS.

\section{Introduction}

With advances in early detection and therapeutics, the cancer survival rate continues to rise worldwide. Cancer survivors, nevertheless, are susceptible to cardiovascular diseases and cardiotoxicity of cancer drugs $[1,2]$. Cardiovascular disorder is a major cause of morbidity and mortality in cancer survivors [3]. These patients are prone to various types of arrhythmias, including tachycardias, bradycardias and conduction disorders [4, 5]. Sometimes cardiac arrhythmias can be life-threatening [6]. In this regard, QT prolongation caused by acquired

(c) The Author(s). 2020 Open Access This article is distributed under the terms of the Creative Commons Attribution 4.0 International License (http://creativecommons.org/licenses/by/4.0/), which permits unrestricted use, distribution, and 
factors associated with cancer therapeutics, termed Acquired Long QT Syndrome (ALQTS), is a key risk state that has been long overlooked in both cardiology and oncology. Using the Pro-QTc score in an institutionwide QT alert system could help identify the high-risk of mortality with markedly prolonged QT interval $(\mathrm{QTc} \geq$ $500 \mathrm{~ms}$ ). In 2017, our group reported cancer to be one of the major factors associated with all-cause mortality in ALQTS [7]. Thus, this study aimed to investigate the impact of ALQTS on the prognosis in hospitalized cancer patients. A priori, we hypothesized that the outcome of cancer patients with ALQTS would be poor, affected by various clinical factors.

\section{Materials and methods}

\section{Study population}

In this matched case-control study, we retrospectively enrolled hospitalized cancer patients with severe ALQTS from September 2013 to April 2016 in the First Affiliated Hospital of Dalian Medical University. Study population was separated into ALQTS group and control group. The inclusion criteria for the ALQTS group were: Hospitalized cancer patients (age $>18$ years) from Hematology-Oncology or other departments showing QTc (Bazett's) $\geq 500 \mathrm{~ms}$ without a family history of inherited LQTS, unexplained syncope, cardiac arrest, or sudden death. The exclusion criteria included QRS duration $>120 \mathrm{~ms}$, presence of complete left or right bundle branch blocks, intraventricular conduction delays or ventricular pacing. Moreover, ECG showing atrial fibrillation/flutter, second-degree and complete atrialventricular blocks, severely sinus, atrial and ventricular tachyarrhythmia, and acute coronary syndrome with dynamic ST-T that interfered accurate QT assessment were excluded. The inclusion criteria for the control group were same as the ALQTS group, except all had a normal QT interval $(350 \mathrm{~ms}<\mathrm{QTc} \leq 440 \mathrm{~ms})$ during hospitalization. The age, sex and the cancer diagnosis were comparable between ALQTS and control groups. This study was approved by the Ethics Committee of the First Affiliated Hospital of Dalian Medical University.

\section{ECG evaluation}

According to the inclusion and exclusion criteria, we retrieved electronic records of standard resting 12-lead ECG parameters from the Muse System (7.1.1 edition), including heart rate (HR) and QTc. ECG were recorded with a paper speed of $25 \mathrm{~mm} / \mathrm{s}$ and voltage of $10 \mathrm{~mm} / 1$ $\mathrm{mV}$ in the supine position, using GE Healthcare MAC 5500 ECG diagnosis systems (India). If differences in the QTc interval exceeded $20 \mathrm{~ms}$ between the machine and hand measurement, the interval was corrected manually, especially in cases with complex $\mathrm{T}$ wave morphologies. In these cases, QT intervals were measured by an experienced QT investigator from lead II, V5 or the lead with the longest QT interval. If several ECG recordings during hospitalization were available, the longest QTc was selected.

\section{Clinical evaluation}

From the electronic medical records, we retrieved data including cancer diagnosis, classification and staging, medical history, chemotherapy drugs and presence of QT-prolonging factors such as electrolyte disorders and use of QT-prolonging drugs. Laboratory tests taken within $48 \mathrm{~h}$ before or after ECG recording were evaluated, including serum potassium (normal range 3.5-5.3 $\mathrm{mmol} / \mathrm{L})$ and serum calcium $(2.02-2.6 \mathrm{mmol} / \mathrm{L})$. QTprolonging drugs used within 7 days of the ECG evaluated in this study were screened through the website (https://www.crediblemeds.org).

\section{Follow-up}

In this study, all-cause death was determined as research endpoint. Telephone survey was conducted after permission obtained from study participants or their legally authorized representatives. When death had occurred, the possible causes were investigated.

\section{Application of the pro-QTc score system}

We employed the Pro-QTc Score to explore the risk of death in patients with QTc $\geq 500 \mathrm{~ms}$. QT-prolonging diagnoses and conditions include cardiomyopathy, acute coronary syndrome, prolonged QT interval, congestive heart failure, bradycardia, diabetes mellitus, stroke, hypokalemia, hypocalcemia, hypomagnesemia, female gender, old age, and several QT-prolonging drugs, which have been compiled in the QT drug list by the Arizona Center for Education and Research on Therapeutics (AZCERT). Each measure in the score system was scaled as 1 point [8]. Pro-QTc scores $\geq 4$ were considered predictive of high mortality risk.

\section{Statistical analyses}

Continuous data were expressed as mean $\pm \mathrm{SD}$, and comparative analysis was performed by Student's $t$-tests for independent samples. The Mann-Whitney $U$ Test was applied when variables were non-normal distributions. Categorical variables were expressed as absolute (n) and relative proportions (\%), and were analyzed using the $X^{2}$ (Chi-square) test. 2-tailed statistical significance was considered when $p<0.05$. Multivariate logistic regression was performed to calculate odds ratios (OR) for various predictors of QT prolongation. For this procedure, variables were selected on the following based on clinical relevance and presence of $p$ values $<0.1$ in univariate analysis. The univariate cumulative probability of all-cause mortality during hospital stay and up to 2-year 
follow-up was assessed by the Kaplan-Meier survival curve using Log-rank statistics. The Cox proportional hazard survival model was used to evaluate the effect of clinical factors on the endpoint. Statistical analyses were performed using SPSS software (version 22.0, SPSS Inc., Chicago, IL).

\section{Results}

Incidence of severe ALQTS in hospitalized cancer patients and their clinical characteristics

Between September 2013 and April 2016, we identified 200 subjects with ALQTS from a total of 10,180 cancer patients hospitalized in the hematology-oncology departments. After applied exclusion criteria and manually checking QT measurement performed by experienced QT investigators, 150 ALQTS patients remained in the study. Compared with age, sex, and cancer type matched 293 non-ALQTS patients, the incidence of hospitalized cancer patients with severe ALQTS was 1.5\% (150/10180).

As to the cancer diagnosis in this study cohort, hematological cancer accounted for $25 \%$, lung cancer $21 \%$, gastrointestinal cancer $20 \%$, breast cancer $17 \%$, gynecologic cancer $7 \%$, urological cancer $3 \%$, prostate cancer $1 \%$, and other cancers $5 \%$. The baseline characteristics of the ALQTS and non-ALQTS groups was summarized in Table 1.

\section{Outcomes and risk factors for QT-prolongation in} hospitalized cancer patients with severe ALQTS

Kaplan-Meier analysis shows that cancer patients with severe ALQTS had much higher all-cause mortality (Fig. 1) compared to age-, sex- and cancer diagnosismatched hospitalized cancer patients with normal QT intervals $(63.3 \%$ vs. $33.4 \%, p<0.001)$. Clinical factors associated with QT prolongation in our study cohort are shown in Table 2.

\section{QT-prolongation and chemotherapeutic drugs}

As shown in Tables 1,31.3\% of patients in the ALQTS group used QT-prolonging drugs, including diphenhydramine $(26 \%)$, famotidine (23\%), quinolone antibiotics (21\%), and furosemide (19\%). Additionally, there were no significant differences among chemotherapeutic drugs between the ALQTS and non-ALQTS groups.

\section{Clinical characteristics and all-cause mortality contributors between non-survivors and survivors in hospitalized cancer patients with severe ALQTS}

Comparisons between survivors and non-survivors in the ALQTS group are presented in Table 3. Multivariable Cox regression was performed between nonsurvivors and survivors in hospitalized cancer patients with severe ALQTS (Table 4). The Cox proportional hazard survival model revealed that male (HR 1.643, 95\%CI 1.023-2.640, $p=0.040$ ), renal insufficiency (HR
Table 1 Clinical characteristics in hospitalized malignant cancer patients with and without severe ALQTS

\begin{tabular}{|c|c|c|c|}
\hline ECG and clinical aspects & $\begin{array}{l}\text { ALQTS } \\
(n=150)\end{array}$ & $\begin{array}{l}\text { Non-ALQTS } \\
(n=293)\end{array}$ & $p$ value \\
\hline Age (years) & $62 \pm 13$ & $62 \pm 13$ & 0.331 \\
\hline \multicolumn{4}{|l|}{ Gender } \\
\hline Female & $58 \%$ & $58 \%$ & \multirow[t]{2}{*}{0.887} \\
\hline Male & $42 \%$ & $42 \%$ & \\
\hline \multicolumn{4}{|l|}{ Comorbidities } \\
\hline Hypertension & $29.3 \%$ & $16.4 \%$ & 0.001 \\
\hline Heart Failure & $6.7 \%$ & $0.7 \%$ & $<0.001$ \\
\hline Arrhythmia & $6.7 \%$ & $2.7 \%$ & 0.047 \\
\hline Renal Insufficiency & $5.3 \%$ & $2.7 \%$ & 0.165 \\
\hline Infection & $21.3 \%$ & $5.1 \%$ & $<0.001$ \\
\hline Type 2 mellitus & $16.0 \%$ & $9.6 \%$ & 0.046 \\
\hline Neurologic diseases & $10.7 \%$ & $3.4 \%$ & 0.002 \\
\hline Anemia & $12.0 \%$ & $1.4 \%$ & $<0.001$ \\
\hline \multicolumn{4}{|l|}{ ECG Data } \\
\hline Heart rate (bpm) & $88 \pm 17$ & $72 \pm 12$ & $<0.001$ \\
\hline $\mathrm{QTc}(\mathrm{ms}) \varphi$ & $520(20)$ & $422(18)$ & $<0.001$ \\
\hline \multicolumn{4}{|l|}{ Laboratory Test } \\
\hline Hypokalemia & $31.7 \%$ & $5.2 \%$ & $<0.001$ \\
\hline Hypocalcemia & $22.7 \%$ & $4.9 \%$ & $<0.001$ \\
\hline \multicolumn{4}{|l|}{ Medications } \\
\hline QT-prolonging drugs & $31.3 \%$ & $6.8 \%$ & $<0.001$ \\
\hline \multicolumn{4}{|l|}{ Chemotherapeutic drugs } \\
\hline Anti-microtubule agents & $36 \%$ & $28 \%$ & 0.084 \\
\hline Platinum & $34 \%$ & $31.1 \%$ & 0.530 \\
\hline Antimetabolites & $33.3 \%$ & $27.3 \%$ & 0.187 \\
\hline Alkylating agents & $29.1 \%$ & $17.7 \%$ & 0.215 \\
\hline Anthracyclines & $28 \%$ & $28.1 \%$ & 0.151 \\
\hline Arsenic trioxide & $2 \%$ & $1.4 \%$ & 0.612 \\
\hline
\end{tabular}

Values are expressed as mean \pm SD or median (interquartile range) unless otherwise indicated. $\varphi$ Median (inter-quartile range) is shown. Abbreviations: ALQTS acquired long QT syndrome, ECG electrocardiograph

5.358, 95\%CI 1.984-14.473, $p=0.001$ ), and hypokalemia (HR 2.315, 95\%CI 1.417-3.781, $p=0.001$ ) were major contributors to all-cause mortality.

\section{Application of the pro-QTc score}

In our study, we applied the Pro-QTc score, proposed by the Mayo Clinic. Figure 2 shows the Pro-QTc scores of patients with severe ALQTS. A significant difference was found between non-survivors and survivors in the group with severe ALQTS group in univariate Cox hazard survival model; however, no difference was ascertained after multivariate adjustment (Table 4). 


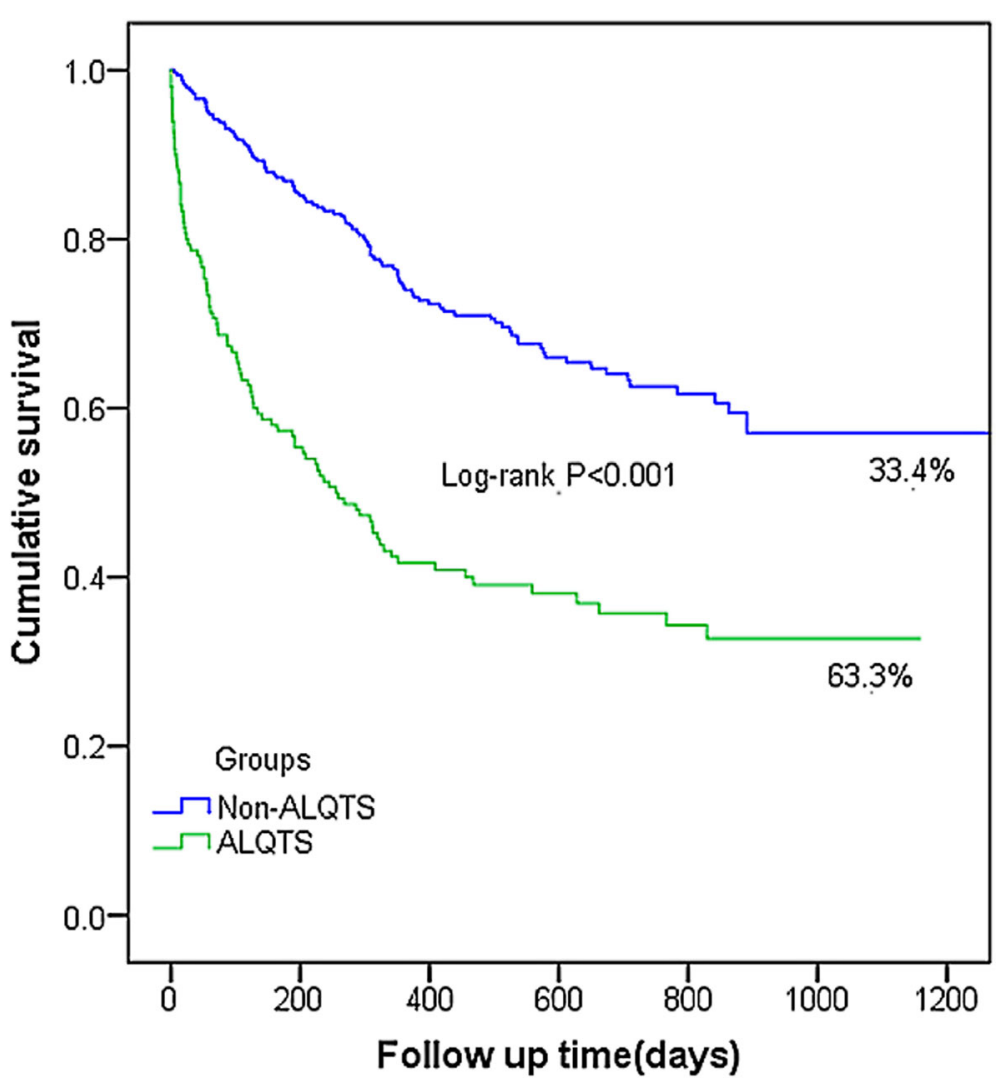

Fig. 1 Kaplan-Meier Survival Curve in Hospitalized Patients with and without Severe ALQTS. Kaplan-Meier analysis shows that cancer patients with severe ALQTS had much higher all-cause mortality. Abbreviations: ALQTS, acquired long QT syndrome

\section{Discussion}

In this study, we found that severe ALQTS (QTc $\geq 500$ $\mathrm{ms})$ accounts for $1.5 \%$ of cancer patients for in-hospital treatment. Among cancer patients, the all-cause mortality is significantly higher in those with ALQTS than those without QTc prolongation. Generally, ALQTS subjects are more severe than those with normal QTc interval (Table 1), with higher frequency of electrolyte imbalances, more use of QT-prolonging drugs and more

Table 2 Clinical factors associated with QT prolongation in hospitalized cancer patients*

\begin{tabular}{lll}
\hline Variables & OR $(95 \% \mathrm{Cl})$ & $p$ value \\
\hline Hypertension & $2.433(1.319-4.489)$ & 0.004 \\
Infection & $3.456(1.471-8.121)$ & 0.004 \\
Anemia & $4.879(1.325-17.961)$ & 0.017 \\
Hypokalemia & $6.271(3.007-13.080)$ & $<0.001$ \\
Hypocalcemia & $2.360(1.028-5.445)$ & 0.043 \\
QT-prolonging drugs & $5.083(2.544-10.155)$ & $<0.001$ \\
Anti-microtubule agents & $2.857(1.658-4.925)$ & $<0.001$
\end{tabular}

*Using multiple logistic regression analysis. Abbreviations: $O R$ odds ratio, $C l$ confidence interval
Table 3 Clinical characteristics in hospitalized cancer patients with severe ALQTS between Non-survivors and survivors

\begin{tabular}{llll}
\hline Parameters & $\begin{array}{l}\text { Non-survivors } \\
(n=95)\end{array}$ & $\begin{array}{l}\text { Survivors } \\
(n=55)\end{array}$ & $P$ Value \\
\hline Age, years & $66 \pm 10$ & $56 \pm 14$ & 0.000 \\
$\quad \geq 70$ years & $36.8 \%$ & $18.2 \%$ & 0.016 \\
Gender & & & \\
$\quad$ Female & $49.5 \%$ & $72.7 \%$ & 0.005 \\
$\quad$ Male & $50.5 \%$ & $27.3 \%$ & \\
HR, bpm & $90 \pm 19$ & $85 \pm 12$ & 0.062 \\
QTc, ms & 513 & 509 & 0.243 \\
Hypokalemia & $40.4 \%$ & $17 \%$ & 0.004 \\
Hypocalcaemia & $26.1 \%$ & $17 \%$ & 0.209 \\
Use of QT-prolonging drugs & $36.4 \%$ & $28.4 \%$ & 0.312 \\
Renal insufficiency & $8.4 \%$ & $0.0 \%$ & 0.027 \\
Infection & $26.3 \%$ & $12.7 \%$ & 0.05 \\
Anemia & $15.8 \%$ & $5.5 \%$ & 0.061 \\
Arrhythmia & $5.3 \%$ & $9.1 \%$ & 0.365 \\
Heart Failure & $7.4 \%$ & $5.5 \%$ & 0.651 \\
Hypertension & $29.5 \%$ & $29.1 \%$ & 0.96 \\
\hline A & &
\end{tabular}

Abbreviations: ALQTS acquired long QT syndrome, HR heart rate 
Table 4 Cox proportional hazard survival between non-survivors and survivors in the severe ALQTS group

\begin{tabular}{|c|c|c|c|c|}
\hline & \multicolumn{2}{|l|}{ Univariate analysis } & \multicolumn{2}{|l|}{ Multivariate analysis } \\
\hline & $\mathrm{HR}(95 \% \mathrm{Cl})$ & $P$ value & $\mathrm{HR}(95 \% \mathrm{Cl})$ & $P$ value \\
\hline Male & $1.793(1.198-2.682)$ & 0.005 & $1.643(1.023-2.640)$ & 0.040 \\
\hline Age $\geq 70$ years & $1.595(1.051-2.420)$ & 0.028 & $1.034(0.624-1.715)$ & 0.896 \\
\hline Pro-QTc $\geq 4$ score & $1.839(1.155-2.929)$ & 0.010 & $1.090(0.561-2.116)$ & 0.799 \\
\hline Hypokalemia & $2.403(1.565-3.689)$ & $<0.001$ & $2.315(1.417-3.781)$ & 0.001 \\
\hline Hypocalcemia & $1.833(1.136-2.959)$ & 0.013 & $1.258(0.697-2.272)$ & 0.446 \\
\hline Renal failure & $4.405(2.097-9.252)$ & $<0.001$ & $5.358(1.984-14.473)$ & 0.001 \\
\hline Infection & $1.970(1.243-3.123)$ & 0.004 & $1.423(0.854-2.373)$ & 0.176 \\
\hline Anemia & $2.388(1.367-4.169)$ & 0.002 & $1.717(0.837-3.523)$ & 0.140 \\
\hline
\end{tabular}

Abbreviations: ALQTS acquired long QT syndrome, HR Hazard Ratio, Cl confidence interval

patients suffering from cancer-related infections, anemia, and concomitant diseases.

Severe ALQTS in hospitalized patients is seen $0.7 \%$ as we reported previously [7]. The prevalence of severe ALQTS in cancer patients revealed in this study is 2-fold (1.5\%) higher indicating delayed ventricular repolarization is much more profound among cancer patients. This finding, however, does not necessarily mean that cancer is a direct cause of QT prolongation. The higher prevalence of ALQTS in cancer patients is likely contributed by 1) presence of QT-prolonging electrolyte imbalance such as hypokalemia and hypocalcaemia (Table 1); 2) use of QT-prolonging drugs; 3) baseline conditions such as hypertension. Most of cancer patients were elders and many had a history of hypertension. Left ventricular hypertrophy resulted from hypertension is a major cause of ALQTS $[9,10]$. Chemotherapy can impair immune system. Thus, post-chemotherapy patients are prone to infections. Infection with inflammatory environment can prolong QT interval due to the effect of

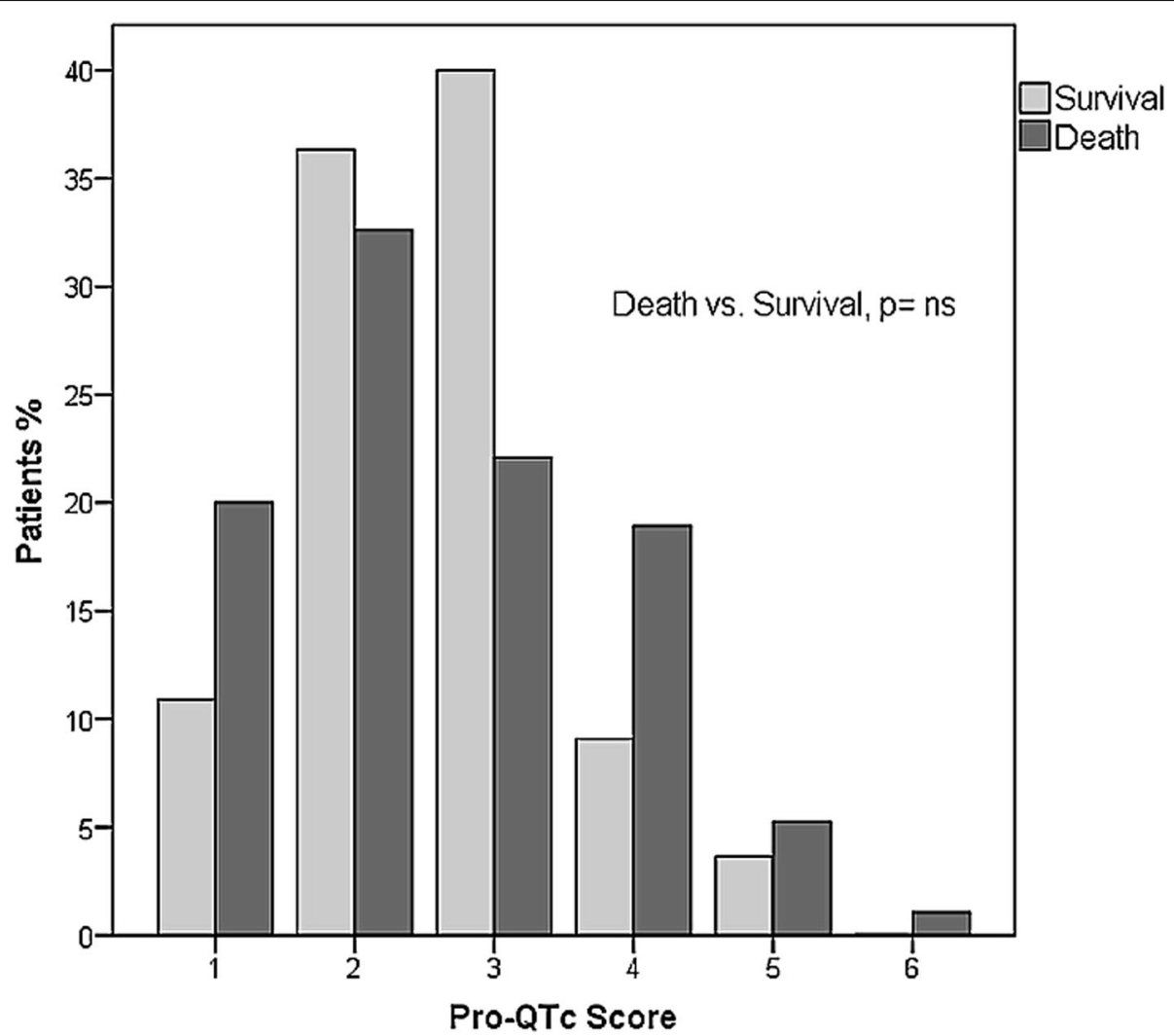

Fig. 2 Pro-QTC score in patients with severe ALQTS. There were no significant/statistical difference between two groups. Abbreviations: ALQTS, acquired long QT syndrome 
inflammatory cytokines (interleukin-6, tumor necrosis factor- $\alpha$, interleukin-1) on potassium and calcium channels [11-14]. QT-prolonging antibiotics such as quinolone antibiotics are commonly prescribed in cancer patients. Anemia is very common in cancers patients especially in late stage. The decreased hemoglobin level can cause tachycardia-cardiomyopathy and increased myocardium mass is associated with QT prolongation [15-17].

As demonstrated in Table 1 cancer patients with severe ALQTS are generally sicker than those without ALQTS. Thus, it is in no surprise that they had a poor outcome measured by a significantly higher all-cause mortality (Fig. 1). In this study, we found that renal insufficiency is one of the contributors to all-cause mortality in cancer patients with ALQTS. Hypokalemia is common in patients with renal insufficiency immediately post dialysis and in patients using potassium-loss diuretics. Therefore, on the basis of previous and our study, we speculate that eliminating the use of QTprolonging medications and monitoring modifiable QTprolonging risk factors such as management of electrolyte disturbance are beneficial for prognosis especially in cancer patients with severe ALQTS.

The application of chemotherapeutic drugs is indispensible and complex in cancer patients. Anthracyclines (doxorubicin) [18], arsenic trioxide [19] vandetanib [6, 20] and molecular-targeted drugs [21-24] have been confirmed to prolong the QTc interval. As shown in Table 1, our study showed that, except for anti-microtubule agents which has been reported to be linked to QTc prolongation $[25,26]$, the rest of chemotherapeutic QT-prolonging drugs are not statistically significant in our study. The insignificance may be related to the small number of patients in terms of each cancer drug used.

In this study, Pro-QTc score $\geq 4$ [7] failed to predict the all-cause mortality in cancer patients. It is perhaps that Pro-QTc was designed to pick up risk factors of sudden arrhythmic death. Most of our non-survival cancer patients died of cancer itself. Prolonged QT interval may reflect the disease severity rather than the direct cause of death in most of cancer non-survivors. Nevertheless, most of QT-prolonging factors are modifiable. Further study is warranted to determine whether eliminating certain risk factors exerts influence on patient outcomes. Establishing an accurate evaluation model to predict mortality risk in cancer patients with ALQTS is urgent.

\section{Limitation}

Our findings should be interpreted in the context of the following limitation. Study outcomes were defined as allcause mortality in this retrospective study, with limited electrophysiological data after patients discharged from hospital. Because of the use of telephone follow-up, specific causes of death cannot be obtained and data analysis related to cardiovascular death is lacking. Further studies are required to examine the effect of ALQTS on the sudden death in hospitalized cancer patients.

\section{Conclusions}

Markedly prolonged QTc interval was seen in $1.5 \%$ of hospitalized cancer patients. The all-cause mortality was high in cancer patients with severe ALQTS.

\section{Abbreviations}

ALQTS: Acquired long QT syndrome; Cl: Confidence interval; HR: Heart rate/ Hazard ratio; OR: Odds ratio

\section{Acknowledgements}

We thank Dr. Li Hong, Dr. Xiaojie Pan and all physicians involved in the ECG analysis and diagnosis of the study subjects.

\section{Authors' contributions}

YL and $\mathrm{HY}$ had full access to all of the data in the study and take responsibility for the integrity of the data and the accuracy of the data analysis; Study concept and design: $\mathrm{YL}$ and $\mathrm{HY}$ and $\mathrm{YX}$; Acquisition, analysis, or interpretation of data: All authors; Drafting of the manuscript: YL and All authors; Critical revision of the manuscript for important intellectual content: $L Z$ and $Y X$; Statistical analysis: YL; Study supervision: $L Z$ and YX. All authors read and approved the final manuscript.

\section{Funding}

This work was supported by the Chang Jiang Scholars Program (T2017124) and Liaoning Distinguished Professor Project (Grant No. [2016]198).

\section{Availability of data and materials}

The datasets used and/or analyses during the current study are available from the corresponding author on reasonable request.

\section{Ethics approval and consent to participate}

This investigation was approved by institutional review board of the First Affiliated Hospital of Dalian Medical University, Dalian, Liaoning, China. The study was conducted according to the Guidelines of the Declaration of Helsinki.

\section{Consent for publication}

The manuscript does not contain any individual person's data in any form.

\section{Competing interests}

The authors declare that they have no competing interests.

\section{Author details}

'Department of Cardiology, First Affiliated Hospital of Dalian Medical University, Zhongshan Road 222, PO Box 116011, Dalian, Liaoning, China. ${ }^{2}$ Department of Cardiology and Institute of Vascular Medicine, Peking University Third Hospital, Beijing, China. ${ }^{3}$ Arrhythmia Center, Ningbo First Hospital, Ningbo, Zhejiang, China. ${ }^{4}$ Sidney Kimmel Medical College of Thomas Jefferson University, Philadelphia, PA, USA. ${ }^{5}$ Lankenau Institute for Medical Research, 100 Lancaster Avenue, Wynnewood, PA 19096, USA.

Received: 12 December 2019 Accepted: 3 February 2020

Published online: 14 February 2020

\section{References}

1. Ferlay J, Steliarova-Foucher E, Lortet-Tieulent J, Rosso S, Coebergh JW, Comber $\mathrm{H}$, et al. Cancer incidence and mortality patterns in Europe: estimates for 40 countries in 2012. Eur J Cancer. 2013;49(6):1374-403.

2. Siegel R, DeSantis C, Virgo K, Stein K, Mariotto A, Smith T, et al. Cancer treatment and survivorship statistics, 2012. CA Cancer J Clin. 2012;62(4): 220-41

3. Ewer MS, Ewer SM. Cardiotoxicity of anticancer treatments. Nat Rev Cardiol. 2015;12(11):620

4. Tamargo J, Caballero R, Delpon E. Cancer chemotherapy and cardiac arrhythmias: a review. Drug Saf. 2015;38(2):129-52. 
5. Yeh ET, Bickford CL. Cardiovascular complications of cancer therapy: incidence, pathogenesis, diagnosis, and management. J Am Coll Cardiol. 2009;53(24):2231-47.

6. Zamorano JL, Lancellotti P, Rodriguez Munoz D, Aboyans V, Asteggiano R, Galderisi M, et al. 2016 ESC position paper on cancer treatments and cardiovascular toxicity developed under the auspices of the ESC Committee for practice guidelines: the task force for cancer treatments and cardiovascular toxicity of the European Society of Cardiology (ESC). Eur Heart J. 2016;37(36):2768-801.

7. Yu H, Zhang L, Liu J, Liu Y, Kowey PR, Zhang Y, et al. Acquired long QT syndrome in hospitalized patients. Heart Rhythm. 2017;14(7):974-8.

8. Haugaa KH, Bos JM, Tarrell RF, Morlan BW, Caraballo PJ, Ackerman MJ Institution-wide QT alert system identifies patients with a high risk of mortality. Mayo Clin Proc. 2013;88(4):315-25.

9. Akintunde AA, Oyedeji AT, Familoni OB, Ayodele OE, Opadijo OG. QT interval prolongation and dispersion: epidemiology and clinical correlates in subjects with newly diagnosed systemic hypertension in Nigeria. J Cardiovasc Dis Res. 2012;3(4):290-5.

10. Mozos I, Serban C. The relation between QT interval and T-wave variables in hypertensive patients. J Pharm Bioallied Sci. 2011;3(3):339-44.

11. London B, Baker LC, Lee JS, Shusterman V, Choi BR, Kubota T, et al. Calciumdependent arrhythmias in transgenic mice with heart failure. Am J Physiol Heart Circ Physiol. 2003;284(2):H431-41.

12. Petkova-Kirova PS, Gursoy E, Mehdi H, McTiernan CF, London B, Salama G. Electrical remodeling of cardiac myocytes from mice with heart failure due to the overexpression of tumor necrosis factor-alpha. Am J Physiol Heart Circ Physiol. 2006;290(5):H2098-107.

13. Fernandez-Velasco M, Ruiz-Hurtado G, Hurtado O, Moro MA, Delgado C. TNF-alpha downregulates transient outward potassium current in rat ventricular myocytes through iNOS overexpression and oxidant species generation. Am J Physiol Heart Circ Physiol. 2007;293(1):H238-45.

14. Wang J, Wang H, Zhang Y, Gao H, Nattel S, Wang Z. Impairment of HERG $\mathrm{K}(+)$ channel function by tumor necrosis factor-alpha: role of reactive oxygen species as a mediator. J Biol Chem. 2004;279(14):13289-92.

15. Upadhya B, Ntim W, Brandon Stacey R, Henderson R, Leedy D, O'Brien FX, et al. Prolongation of QTc intervals and risk of death among patients with sickle cell disease. Eur J Haematol. 2013:91(2):170-8.

16. Liem Rl, Young LT, Thompson AA. Prolonged QTC interval in children and young adults with sickle cell disease at steady state. Pediatr Blood Cancer. 2009;52(7):842-6

17. Fei YD, Li YG, Surkis W, Zhang L. Does Anemia cause QT prolongation in patients with hematologic disorders? Chin Med J. 2015;128(24):3385-6.

18. Ducroq J, Moha ou Maati H, Guilbot S, Dilly S, Laemmel E, Pons-Himbert C, et al. Dexrazoxane protects the heart from acute doxorubicin-induced QT prolongation: a key role for I(Ks). Br J Pharmacol. 2010;159(1):93-101.

19. Barbey JT, Pezzullo JC, Soignet SL. Effect of arsenic trioxide on QT interval in patients with advanced malignancies. J Clin Oncol. 2003;21(19):3609-15.

20. Wells SA Jr, Gosnell JE, Gagel RF, Moley J, Pfister D, Sosa JA, et al. Vandetanib for the treatment of patients with locally advanced or metastatic hereditary medullary thyroid cancer. J Clin Oncol. 2010;28(5): 767-72.

21. Lenihan DJ, Kowey PR. Overview and management of cardiac adverse events associated with tyrosine kinase inhibitors. Oncologist. 2013;18(8): 900-8.

22. Schmidinger M, Zielinski CC, Vogl UM, Bojic A, Bojic M, Schukro C, et al. Cardiac toxicity of sunitinib and sorafenib in patients with metastatic renal cell carcinoma. J Clin Oncol. 2008;26(32):5204-12.

23. Shah RR, Morganroth J, Shah DR. Cardiovascular safety of tyrosine kinase inhibitors: with a special focus on cardiac repolarisation (QT interval). Drug Saf. 2013;36(5):295-316.

24. Strevel EL, Ing DJ, Siu LL. Molecularly targeted oncology therapeutics and prolongation of the QT interval. J Clin Oncol. 2007;25(22):3362-71.

25. Arbuck SG, Strauss H, Rowinsky E, Christian M, Suffness M, Adams J, et al. A reassessment of cardiac toxicity associated with Taxol. J Natl Cancer Inst Monogr. 1993;15:117-30.

26. Kamineni P, Prakasa K, Hasan SP, Akula R, Dawkins F. Cardiotoxicities of paclitaxel in African Americans. J Natl Med Assoc. 2003;95(10):977-81.

\section{Publisher's Note}

Springer Nature remains neutral with regard to jurisdictional claims in published maps and institutional affiliations.

Ready to submit your research? Choose BMC and benefit from:

- fast, convenient online submission

- thorough peer review by experienced researchers in your field

- rapid publication on acceptance

- support for research data, including large and complex data types

- gold Open Access which fosters wider collaboration and increased citations

- maximum visibility for your research: over $100 \mathrm{M}$ website views per year

At $\mathrm{BMC}$, research is always in progress.

Learn more biomedcentral.com/submissions 